\title{
CARACTERIZACIÓN DE LA FLORA EPÍFITA VASCULAR DEL PARQUE IBEROAMÉRICA, SANTO DOMINGO, REPÚBLICA DOMINICANA
}

\section{Characterization of the vascular epiphyte flora of the Iberoamérica Park, Santo Domingo, Dominican Republic}

\author{
Cristopher Jiménez-Orozco \\ Universidad Autónoma de Santo Domingo \\ (UASD), Escuela de Biología, Santo Domingo, \\ República Dominicana. Instituto de Investigaciones \\ Botánicas y Zoológicas Prof. Rafael M. Moscoso (IIBZ) \\ Correo-e: cristopheruasd@gmail.com \\ Berleni V. Lebrón-Liriano \\ Universidad Autónoma de Santo Domingo \\ (UASD), Escuela de Biología, Santo Domingo, \\ República Dominicana \\ Rosy Fernández-Gutiérrez \\ Universidad Autónoma de Santo Domingo \\ (UASD), Escuela de Biología, Santo Domingo, \\ República Dominicana
}

\author{
Rumarda Urbáez \\ Universidad Autónoma de Santo Domingo \\ (UASD), Escuela de Biología, Santo Domingo, \\ República Dominicana \\ Ángela Guerrero \\ Universidad Autónoma de Santo Domingo \\ (UASD), Escuela de Biología, Santo Domingo, \\ República Dominicana
}

Recibido: 5/5/2019 • Aprobado: 22/5/2019

Cómo citar: Jiménez-Orozco, C., Lebrón-Liriano, B. V., Fernández-Gutiérrez, R., Urbáez, R., \& Guerrero, Ángela. (2019). Caracterización de la flora epífita vascular del Parque Iberoamérica, Santo Domingo, República Dominicana. Ciencia, Ambiente y Clima, 2(1), 23-33. doi: https://doi.org/10.22206/cac.2019.v2i1.pp23-33

\section{Resumen}

Se presentan los resultados del inventario de la flora epífita vascular del Parque Iberoamérica, un área verde de la ciudad de Santo Domingo, República Dominicana. Los muestreos fueron realizados durante un mes, desde el 21 de noviembre hasta el 13 de diciembre del año 2017, utilizando el método de transecto y parcela. El transecto, de 1,200 m de largo x $30 \mathrm{~m}$ de ancho, cubrió el área total del parque $\left(1.2 \mathrm{~km}^{2}\right)$. En el caso de la parcela, el área de ocupación fue menor $\left(120 \mathrm{~m}^{2}\right)$, esta se delimitó en el sitio de mayor cobertura vegetal dentro del parque. Además, se hicieron recorridos fuera del transecto y la parcela para documentar la mayor diversidad posible. Se identificaron 35 especies, distribuidas en 29 géneros y 23 familias. Las

\begin{abstract}
The results of the epiphytic vascular flora inventory carried out in Parque Iberoamérica, an urban green area of Santo Domingo, Dominican Republic, are presented. The samplings were carried out during one month (Nov $21^{\text {st }}-$ Dec $13^{\text {th }}$,2017) using transect and plot design. The transect, of $1,200 \mathrm{~m}$ long $\times 30 \mathrm{~m}$ wide, covered the total area of the park $\left(1,2 \mathrm{~km}^{2}\right)$. In the case of the plot, the occupation area was smaller $\left(120 \mathrm{~m}^{2}\right)$. This is the main page of the vegetation cover within the park. In order to document as much diversity as possible, areas outside the transect and plot were roamed. 35 species were identified, distributed in 29 genera and 23 families. The most representative families were: Moraceae, with 4 species, followed by Bromeliaceae and
\end{abstract}




\section{Cristopher Jiménez-Orozco, Berleni V. Lebrón-Liriano, Rosy Fernández-Gutiérrez, \\ Rumarda Urbáez y Ángela Guerrero}

familias más representativas fueron: Moraceae con cuatro especies, seguida de Bromeliaceae y Fabaceae con tres especies cada una. Las especies más abundantes fueron Tillandsia recurvata con 3,531 individuos, seguida de Tillandsia balbisiana con 556 individuos, y Nephrolepis acutifolia con 296. A pesar del reducido tamaño del parque, en términos de composición florística, la comunidad de epífitas es diversa.

Palabras clave: diversidad; Tillandsia recurvata; Ficus crassinervia; Bromeliaceae; Moraceae.

\section{Introducción}

El epifitismo es una interacción comensalística entre dos especies (el forófito y una planta epífita) (Smith \& Smith, 2001). El forófito es un árbol vivo que brinda soporte a otra especie sin recibir daño fisiológico, mientras que la planta epífita al fijarse en la corteza del tronco, ramas e incluso en las hojas, logra una posición más elevada y de mayor alcance a la luz y precipitación (Benzing, 1990; Nieder, Ibisch, \& Barthlot, 1997).

Las epífitas vasculares son un elemento conspicuo de las comunidades vegetales de los trópicos. Se estima que el $10 \%$ de las plantas vasculares y aproximadamente el $30 \%$ de la diversidad de plantas neotropicales son epífitas (Gentry \& Dodson, 1987; Wolf \& Flamenco, 2003; Wester, et al., 2011). Estas plantas poseen diversos mecanismos para lidiar con la sequía y la falta de nutrientes (Zotz \& Hietz, 2001). Las mismas requieren en ocasiones de interacciones mutualísticas con microorganismos, artrópodos y algunos grupos de vertebrados, además de que exhiben un alto grado de especialización en términos morfoanatómicos (Granados, López, Hernández, \& Sánchez, 2003).

Las epífitas también juegan un papel ecológico crucial para otros niveles tróficos, ya que funcionan como sitios de alimentación, refugio y reproducción para una gran cantidad de organismos principalmente anfibios e insectos. (Gentry \& Dodson, 1987; Greeney, 2001).
Fabaceae with 3 species each. The most abundant species were Tillandsia recurvata with 3,531 individuals, followed by T. balbisiana with 556, and Nephrolepis acutifolia with 296. Despite the reduced area of the park, in terms of floristic composition, the epiphytes community is diverse.

Keywords: Diversity; Tillandsia recurvata; Ficus crassinervia; Bromeliaceae; Moraceae.

En el pasado las plantas epífitas eran un grupo marginado y poco estudiado, debido a que se afirmaba que el aporte de biomasa de las mismas al ecosistema era poco relevante y su muestreo era dificultoso (Bruijnzeel \& Hamilton, 2001; Gradstein, Nadkarni, Krömer, Holz, \& Nöske, 2003). Sin embargo, las investigaciones sobre la ecología y taxonomía de este grupo han aumentado en las últimas décadas, concentrándose principalmente en el Neotrópico (Benzing, 1987; Nieder, Engwald \& Barthlott, 1999; Freiberg \& Freiberg, 2000; Krömer \& Gradstein, 2003; Zotz \& Schultz, 2008).

En República Dominicana los trabajos sobre la riqueza y distribución de la flora epífita vascular son escasos, la mayor parte de la información deriva de inventarios florísticos. Sin embargo, en otros países de la región ya se han realizado estudios de esta naturaleza: Puerto Rico (Ackerman, Montalvo \& Vera, 1989; Migenis \& Ackerman, 1993), Cuba (García-González et al., 2016; Granado \& García, 2016) y Jamaica (Kelly, 1985).

De las pocas investigaciones realizadas en el país cabe mencionar el trabajo de Ruiz, Ortíz y Guerrero (2015), quienes analizaron la distribución y ecología de la familia Bromeliaceae en La Española. En dicho estudio se resalta la importancia de estas plantas como componentes estructurales de las comunidades de epífitas, principalmente en los bosques húmedos y nublados. Las bromelias están distribuidas en toda la isla y el $52 \%$ de las especies 
reportadas son exclusivamente de hábito epífito. El objetivo de esta investigación es determinar la diversidad y composición de la flora epífita vascular del Parque Iberoamérica, un parque urbano de la ciudad de Santo Domingo, República Dominicana.

\section{Materiales y métodos}

\section{1. Área de estudio}

El Parque Iberoamérica pertenece al sistema de áreas verdes de la ciudad de Santo Domingo, República Dominicana. Está localizado entre las Avenidas Pedro Henríquez Ureńa y Simón Bolívar, sobre las coordenadas $18^{\circ} 28^{\prime} 01^{\prime \prime} \mathrm{N}$ y $69^{\circ} 55^{\prime} 06^{\prime \prime} \mathrm{W}$, y tiene una extensión de $1.2 \mathrm{~km}^{2}$ (Figura 1). El clima es subtropical, con estación seca de diciembre a marzo y época de lluvia de mayo a noviembre; la temperatura promedio anual registrada es de $25^{\circ} \mathrm{C}$ y la humedad relativa de $89 \%$; la pluviometría promedio anual es de 1,393 mm (ONAMET, 2019). El relieve es irregular con numerosas ondulaciones y la vegetación se desarrolla sobre un pequeño farallón de roca caliza de origen coralino, del plioceno y pleistoceno (Llinás, 2005).

Esta área, por su topografía de cavernas y arrecifes coralinos, permitió el desarrollo de un característico tipo de vegetación conocido como bosque costero sobre rocas (Hager \& Zanoni, 1993), del cual solo quedan remanentes fragmentarios o relictos. Por eso esta zona albergó durante la dictadura de Rafael Leonidas Trujillo un Parque Zoológico, construido entre 1952-1955 por el Ing. Emile Boyre. En esos terrenos también funcionó Quisqueya Park, uno de los pocos parques de atracciones permanentes de la ciudad de Santo Domingo. El mismo estuvo en funcionamiento en los años 80 hasta 1991. Años posteriores fue conocido como el Parque de las Luces y su infraestructura fue remozada en diciembre de 2012, por el entonces alcalde Roberto Salcedo.

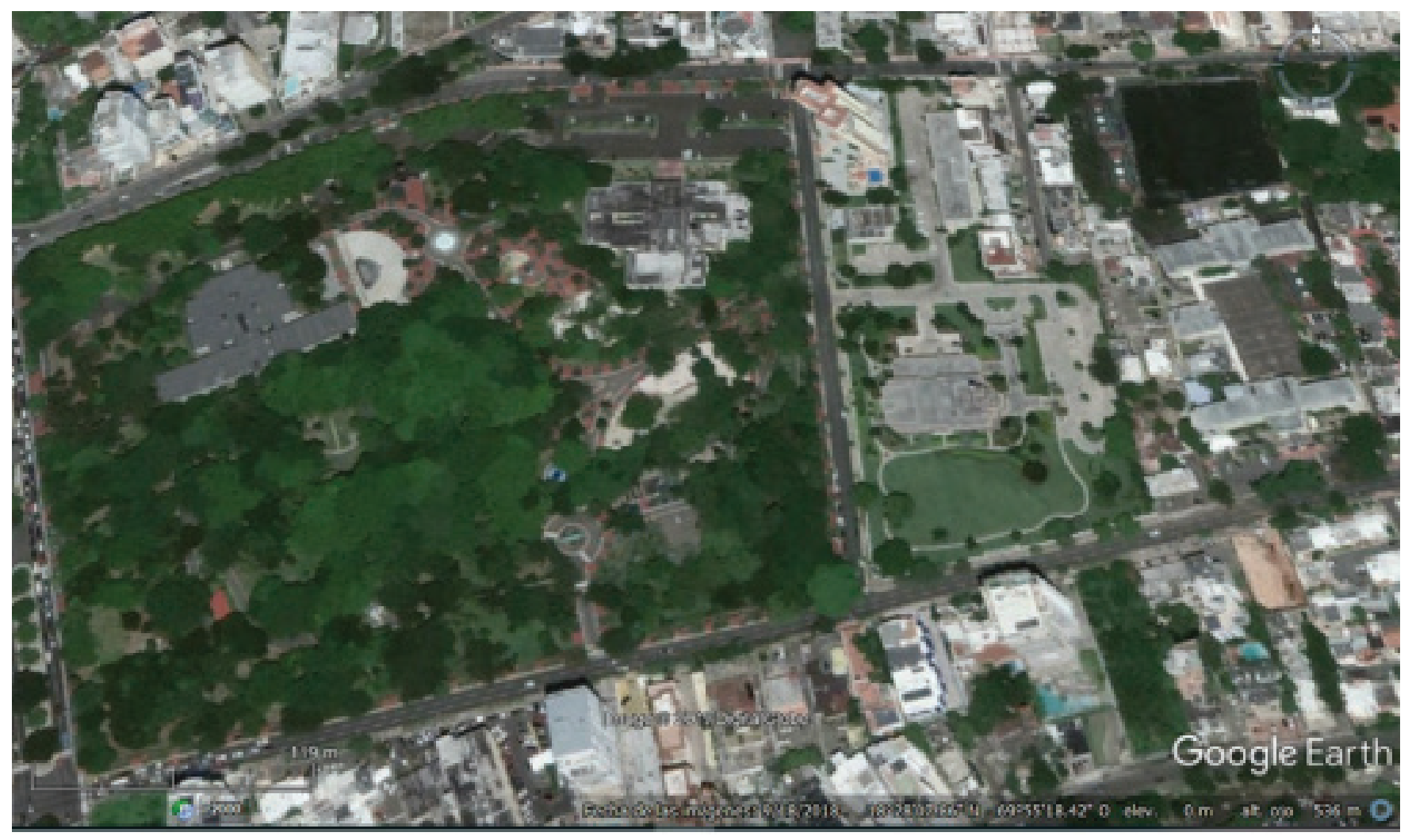

Figura 1. Localización del Parque Iberoamérica. Fuente: elaborado por J. Then. 


\section{Cristopher Jiménez-Orozco, Berleni V. Lebrón-Liriano, Rosy Fernández-Gutiérrez, \\ Rumarda Urbáez y Ángela Guerrero}

\subsection{Equipos y materiales usados}

Los materiales y equipos usados fueron: cinta métrica, relascopio, crayones, libreta de campo y bolígrafos. Para el registro de los datos se diseñó un formulario, en el que se registraron las especies de epífitas, y forófitos. A estos últimos se les midió altura y diámetro a la altura de pecho (DAP).

\subsection{Técnicas de muestreo}

El trabajo de campo se llevó a cabo durante un mes, desde el 21 de noviembre hasta el 13 de diciembre del año 2017. Se delimitó un transecto de 1,200 $\mathrm{m}$ x $30 \mathrm{~m}$ por todo el perímetro del parque y una parcela de $120 \mathrm{~m}^{2}$ en el área de mayor cobertura vegetal, donde se procedió a registrar las especies de epífitas vasculares y su abundancia.

En el caso de los forófitos, solo fueron incluidos en el muestreo los árboles con un DAP mayor a $5 \mathrm{~cm}$ (aproximadamente a $1.5 \mathrm{~m}$ sobre el suelo), excluyendo plantas arbustivas. En la parcela se siguió el mismo procedimiento que en el transecto. Las especies fueron identificadas in situ; aquellas cuya identidad era dudosa fueron colectadas para su posterior identificación utilizando claves taxonómicas y consultas a especialistas. Los nombres científicos fueron verificados en las bases de datos The Plant List (2013) y Tropicos ${ }^{\circledast}$ (2010).

\subsection{Análisis estadísticos}

La diversidad Alfa fue determinada con los índices de diversidad de Shannon (Fórmula 1) y Simpson (Fórmula 2) (Moreno, 2001). Para comparar la similitud florística entre el transecto y la parcela se empleó el Coeficiente de Similitud de Sørensen, el cual está basado en la presencia/ausencia de especies (Krebs, 1999). Los datos fueron corridos en el software Biodiversity Calculator versión 2.0.

$$
\mathrm{H}^{\prime}=-\sum \mathrm{p}_{\mathrm{i}} \ln \mathrm{p}_{\mathrm{i}}
$$

Fórmula 1. Diversidad Alfa, índice de diversidad de Shannon.

En donde:

$$
\begin{aligned}
& \mathrm{H}^{\prime}=\text { índice de Shannon. } \\
& \mathrm{Ln}=\text { logaritmo neperiano. } \\
& \mathrm{Pi}=\text { abundancia proporcional de la especie } \mathrm{i} \\
& \text { es decir, el número de individuos de la especie } \\
& \text { i dividido entre el número total de individuos } \\
& \text { de la muestra. }
\end{aligned}
$$

$$
\lambda=\sum \mathrm{p}_{i}^{2}
$$

Fórmula 2. Diversidad Alfa, índice de diversidad de Simpson.

En donde:

$\mathrm{Pi}=$ número de individuos de la especie $i$ dividido entre el número total de individuos de la comunidad.

\section{Resultados}

\subsection{Composición florística, abundancia y ri- queza de epífitas vasculares}

Se registraron 34 especies de epífitas correspondientes a 29 géneros y 23 familias. La familia más representativa fue Moraceae con cuatro especies, seguida de Bromeliaceae y Fabaceae con tres especies cada una (Tabla 1, Figura 2). El género más diverso fue Ficus con cuatro especies. Se cuantificó un total de 4,665 individuos de epífitas vasculares. Las especies más abundantes fueron Tillandsia recurvata con 3,531 individuos, Tillandsia balbisiana con 556 y Nephrolepis acutifolia con 296 (Figura 2). 
Tabla 1. Especies de plantas epífitas reportadas para el Parque Iberoamérica

\section{Leyenda:}

A = Abundancia relativa

Puntos de muestreo:

$\mathrm{T}=$ Transecto

$\mathrm{P}=$ Parcela

$S$ = Estatus Biogeográfico

$\mathrm{N}=$ Nativa

$\mathrm{E}=$ Exótica

\begin{tabular}{|c|c|c|c|c|}
\hline Especie/Familia & A & $\mathbf{S}$ & $\mathbf{T}$ & $\mathbf{P}$ \\
\hline \multicolumn{5}{|l|}{ PTERIDOPHYTA } \\
\hline \multicolumn{5}{|l|}{ NEPHROLEPIDACEAE } \\
\hline Nephrolepis acutifolia (Desv.) Christ. & 296 & $\mathrm{E}$ & $\mathrm{X}$ & \\
\hline \multicolumn{5}{|l|}{ POLYPODIACEAE } \\
\hline Phlebodium aureum (L.) J. Sm. & 5 & $\mathrm{~N}$ & & $\mathrm{X}$ \\
\hline $\begin{array}{l}\text { Pleopeltis polypodioides (L.) E.G. Andrews \& } \\
\text { Windham. }\end{array}$ & 1 & $\mathrm{~N}$ & $\mathrm{X}$ & \\
\hline \multicolumn{5}{|l|}{ MAGNOLIOPHYTA (ANGIOSPERMAS) } \\
\hline \multicolumn{5}{|l|}{ ARACEAE } \\
\hline Syngonium auritum (L.) Schott & 1 & $\mathrm{~N}$ & & $\mathrm{X}$ \\
\hline \multicolumn{5}{|l|}{ ARECACEAE } \\
\hline Acrocomia aculeata (Jacq.) Lodd. ex Mart. & 3 & $\mathrm{~N}$ & & $\mathrm{X}$ \\
\hline Adonidia merrillii (Becc.) Becc. & 2 & $\mathrm{E}$ & $\mathrm{X}$ & $\mathrm{X}$ \\
\hline Morfoespecie 1 & 23 & & $\mathrm{X}$ & \\
\hline \multicolumn{5}{|l|}{ COMMELINACEAE } \\
\hline Tradescantia spathacea Sw. & 20 & $\mathrm{~N}$ & $\mathrm{X}$ & $\mathrm{X}$ \\
\hline Commelina diffusa Burm.f. & 9 & $\mathrm{~N}$ & & $\mathrm{X}$ \\
\hline \multicolumn{5}{|l|}{ BROMELIACEAE } \\
\hline Neoregelia spectabilis (T. Moore) L. B. Sm. & 1 & $\mathrm{~N}$ & $\mathrm{X}$ & \\
\hline Tillandsia recurvata (L.) L. & 3,531 & $\mathrm{~N}$ & $\mathrm{X}$ & $\mathrm{X}$ \\
\hline Tillandsia balbisiana Schult. \& Schult.f. & 556 & $\mathrm{~N}$ & $\mathrm{X}$ & $\mathrm{X}$ \\
\hline \multicolumn{5}{|l|}{ VITACEAE } \\
\hline Cissus verticillata (L.) Nicolson \& C. E. Jarvis. & 1 & $\mathrm{~N}$ & $\mathrm{X}$ & \\
\hline \multicolumn{5}{|l|}{ FABACEAE } \\
\hline Leucaena leucocephala (Lam.) de Wit. & 1 & $\mathrm{E}$ & $\mathrm{X}$ & \\
\hline Pithecellobium dulce (Roxb.) Benth. & 4 & $\mathrm{E}$ & $\mathrm{X}$ & \\
\hline Delonix regia (Hook.) Raf. & 7 & $\mathrm{E}$ & $\mathrm{X}$ & \\
\hline
\end{tabular}

\begin{tabular}{|c|c|c|c|c|}
\hline Especie/Familia & A & $S$ & $\mathbf{T}$ & $\mathbf{P}$ \\
\hline \multicolumn{5}{|l|}{ MORACEAE } \\
\hline Ficus citrifolia Mill. & 3 & $\mathrm{~N}$ & $\mathrm{X}$ & \\
\hline Ficus microcarpa L.f. & 50 & $\mathrm{E}$ & $\mathrm{X}$ & \\
\hline Ficus crassinervia Desf. ex Willd. & 58 & $\mathrm{~N}$ & $\mathrm{X}$ & \\
\hline Ficus crocata (Miq.) Mart. ex Miq. & 5 & $\mathrm{X}$ & $\mathrm{X}$ & \\
\hline \multicolumn{5}{|l|}{ URTICACEAE } \\
\hline Pilea microphylla (L.) Liebm. & 23 & $\mathrm{~N}$ & $\mathrm{X}$ & \\
\hline \multicolumn{5}{|l|}{ CUCURBITACEAE } \\
\hline Melothria pendula L. & 12 & $\mathrm{~N}$ & $\mathrm{X}$ & \\
\hline \multicolumn{5}{|l|}{ OXALIDACEAE } \\
\hline Oxalis barrelieri $\mathrm{L}$. & 1 & $\mathrm{~N}$ & $\mathrm{X}$ & \\
\hline \multicolumn{5}{|l|}{ EUPHORBIACEAE } \\
\hline Hura crepitans L. & 2 & $\mathrm{E}$ & $\mathrm{X}$ & \\
\hline \multicolumn{5}{|l|}{ PHYLLANTACEAE } \\
\hline Phyllantus niruri $\mathrm{L}$. & 20 & & $\mathrm{X}$ & \\
\hline \multicolumn{5}{|l|}{ COMBRETACEAE } \\
\hline Terminalia catappa $\mathrm{L}$. & 1 & $\mathrm{E}$ & $\mathrm{X}$ & \\
\hline \multicolumn{5}{|l|}{ BURSERACEAE } \\
\hline Bursera simaruba (L.) Sarg. & 1 & $\mathrm{~N}$ & $\mathrm{X}$ & \\
\hline \multicolumn{5}{|l|}{ MELIACEAE } \\
\hline Azadirachta indica A. Juss. & 1 & $\mathrm{E}$ & $\mathrm{X}$ & \\
\hline \multicolumn{5}{|l|}{ MALVACEAE } \\
\hline Sterculia apetala (Jacq.) H. Karst. & 2 & $\mathrm{E}$ & $\mathrm{X}$ & \\
\hline \multicolumn{5}{|l|}{ PETIVERIACEAE } \\
\hline Rivina humilis $\mathrm{L}$. & 1 & $\mathrm{~N}$ & $\mathrm{X}$ & \\
\hline \multicolumn{5}{|l|}{ OLEACEAE } \\
\hline Jasminum fluminense Vell. & 1 & $\mathrm{E}$ & $\mathrm{X}$ & \\
\hline \multicolumn{5}{|l|}{ ACANTHACEAE } \\
\hline Morfoespecie 2 & 4 & & $\mathrm{X}$ & \\
\hline \multicolumn{5}{|l|}{ BIGNONIACEAE } \\
\hline Tabebuia rosea (Bertol.) Bertero ex A. DC. & 1 & $\mathrm{E}$ & $\mathrm{X}$ & \\
\hline \multicolumn{4}{|l|}{ VERBENACEAE } & \\
\hline Priva lappulacea (L.) Pers. & 1 & $\mathrm{~N}$ & $\mathrm{X}$ & \\
\hline \multicolumn{5}{|l|}{ ARALIACEAE } \\
\hline Schefflera actinophylla (Endl.) Harms & 17 & $\mathrm{E}$ & $\mathrm{X}$ & \\
\hline
\end{tabular}




\section{Cristopher Jiménez-Orozco, Berleni V. Lebrón-Liriano, Rosy Fernández-Gutiérrez, \\ Rumarda Urbáez y Ángela Guerrero}

\section{Riqueza de especies de epífitas por familia}

- Commelinaceae

- Fabaceae

- Oleaceae

- Phyllantaceae

- Urticaceae

- Oxalidaceae

- Petiveraceae

- Acanthaceae

- Nephrolepidaceae

- Malvaceae

- Moraceae
- Arecaceae

- Polypodiaceae

- Bignoniaceae

- Cucurbitaceae

- Vitaceae

- Verbenaceae

- Meliaceae

- Burseraceae

- Euphorbiaceae

- Bromeliaceae

- Araliaceae

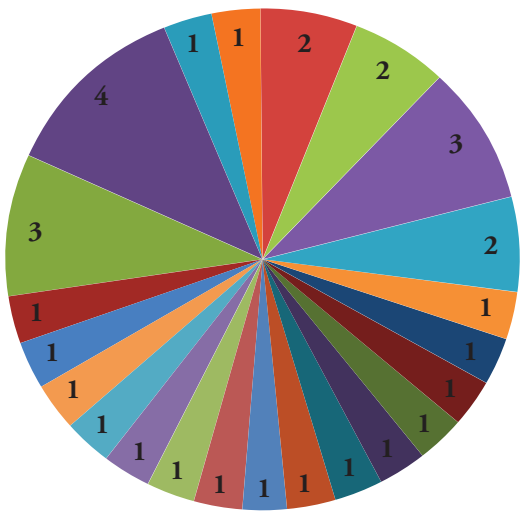

Figura 2. Riqueza de especies de epífitas por familia. Original de Jiménez-Orozco et al.

Las epífitas no mostraron preferencia por forófitos en particular a excepción de Nephrolepis acutifolia (Nephrolepidaceae) que solo se registró sobre Phoenix dactylifera (Arecaceae). Por otro lado, se observó que la especie Delonix regia (Fabaceae) fue el hospedero que albergó a la mayoría de las especies de epífitas (18).

\subsection{Riqueza de forófitos}

Se registran como forófitos 29 especies agrupadas en 29 géneros y 17 familias de angiospermas. La familia más diversa fue Fabaceae con 5 especies (Delonix regia, Haematoxylum campechianum, Hymenaea courbaril, Pithecellobium dulce y Senna siamea), representando un $20 \%$ del total de especies. Se cuantificó un total de 178 individuos, siendo las especies más abundantes Delonix regia (35 individuos), Phoenix dactylifera (17), Citharexylum spinosum (16) y Hura crepitans (10) (Tabla 2, Figura 3).

Tabla 2. Especies de forófitos registradas en el Parque Iberoamérica.

\section{Leyenda:}

ES (especie)

FA (familia)

A (abundancia relativa)

DAP (diámetro a la altura de pecho)

\begin{tabular}{|l|l|l|}
\hline ES/FA & A & DAP $(\mathbf{c m})$ \\
\hline ANACARDIACEAE & & \\
\hline Spondias mombin L. & 17 & $120-161$ \\
\hline ARECACEAE & & \\
\hline Roystonea borinquena O. F.Cook & 2 & $86-100$ \\
\hline Phoenix dactylifera L. & 2 & $187-280$ \\
\hline ANNONACEAE & & \\
\hline Annona reticulata L. & 1 & 16.1 \\
\hline APOCYNACEAE & & \\
\hline Neriun oleander L. & 1 & 14.2 \\
\hline BIGNONIACEAE & & \\
\hline Catalpa longissima (Jacq.) Dum.Cours. & 20 & $14.1-98.3$ \\
\hline Tabebuia rosea (Bertol.) Bertero ex A.DC. & 2 & $28-38.8$ \\
\hline BORRAGINACEAE & & \\
\hline Cordia alliodora (Ruiz \& Pav.) Oken & 1 & $12-128$ \\
\hline BURSERACEAE & & \\
\hline Bursera simaruba (L.) Sarg. & 1 & 139.1 \\
\hline CASUARINACEAE & 5 & $126.2-240$ \\
\hline Casuarina equisetifolia L. & 2 & $46-190$ \\
\hline COMBRETACEAE & 2 & $159.3-175.4$ \\
\hline Terminalia catappa L. & 10 & $150-480$ \\
\hline EUPHORBIACEAE & & \\
\hline Hura crepitans L. & 38 & $100-290$ \\
\hline FABACEAE & & $150-300$ \\
\hline Delonix regia (Hook.) Raf. & & \\
\hline Haematoxylum campechianum L. & & \\
\hline Hymenaea courbaril L. & & \\
\hline Pithecellobium dulce (Roxb.) Benth. & & \\
\hline
\end{tabular}




\begin{tabular}{|l|l|l|}
\hline ES/FA & A & DAP (cm) \\
\hline Senna siamea (Lam.) H. S.Irwin \& Barneby & 2 & $114-138$ \\
\hline LAMIACEAE & & \\
\hline Tectona grandis L.f. & 2 & $179-250$ \\
\hline MALVACEAE & & \\
\hline Ceiba pentandra (L.) Gaertn. & 3 & $49-93$ \\
\hline Sterculia apetala (Jacq.) H. Karst. & 3 & $36-66.1$ \\
\hline MELIACEAE & & \\
\hline Azadirachta indica A. Juss. & 1 & 240 \\
\hline Swietenia mahagoni (L.) Jacq. & 13 & $7-53$ \\
\hline MORACEAE & & \\
\hline Ficus microcarpa L.f. & 1 & 271 \\
\hline Ficus crassinervia Desf. ex Willd. & 1 & 252 \\
\hline POLYGONACEAE & & \\
\hline Coccoloba diversifolia Jacq. & 6 & $12.2-130$ \\
\hline PRIMULACEAE & & \\
\hline Petesiodes clusiifolium (Sw.) Kuntze & 1 & 24.2 \\
\hline SAPINDACEAE & & \\
\hline Melicoccus bijugatus Jacq. & 1 & 81 \\
\hline SAPOTACEAE & & \\
\hline Sideroxylon foetidissimum Jacq. & 1 & 143 \\
\hline VERBENACEAE & 16 & $17.2-67.3$ \\
\hline Citharexylum spinosum L. & & \\
\hline
\end{tabular}

Algunas especies como D. regia, Ficus crassinervia, Roystonea borinquena, Terminalia cattapa, Sterculia apetala, Tabebuia rosea, Bursera simaruba, P. dulce, H.crepitans y Ficus microcarpa se comportaron como forófitos y epífitas, condición que no se registró en otras taxa. Sin embargo, fue más frecuente observarlas como hospederos.

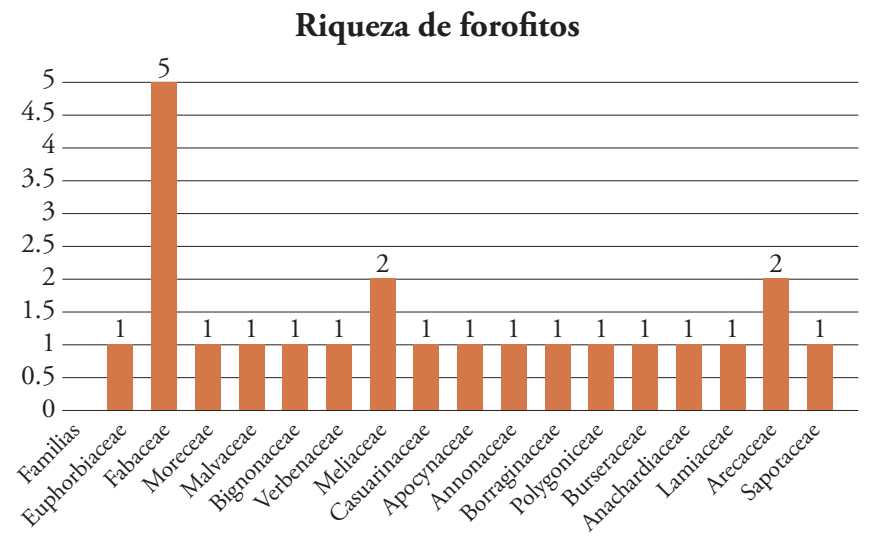

Figura 3. Riqueza de forófitos por familias. Original de Jiménez-Orozco et al.

\subsection{Abundancia de epífitas vs. DAP}

Respecto a la distribución de las epífitas, se observó que los forófitos con menor DAP albergaron una mayor abundancia de epífitas (Figura 4). La especie Swietenia mahagoni (Meliaceae) presentó los diámetros más bajos $(7 \mathrm{~cm}-53 \mathrm{~cm})$ con un total de 319 individuos, principalmente bromelias del género Tillandsia.

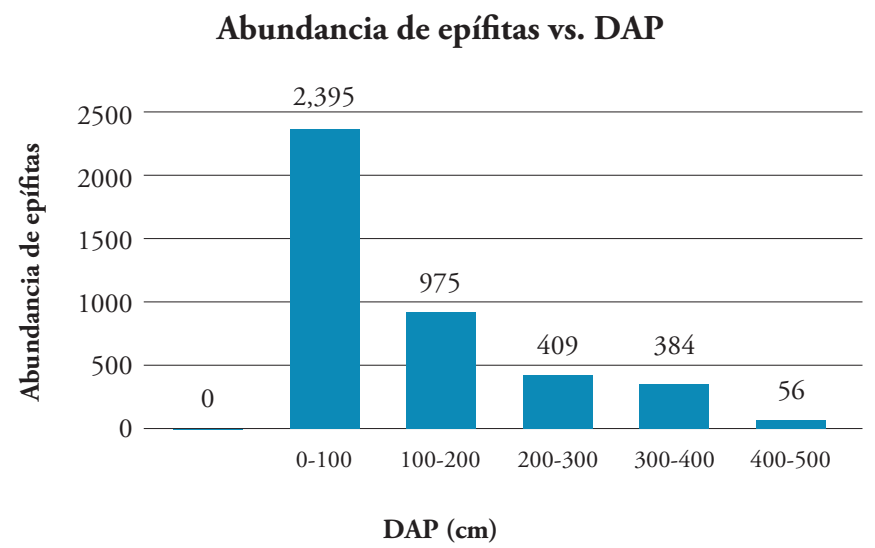

Figura 4. Abundancia de epífitas vs. DAP. Original de Jiménez-Orozco et al.

\subsection{Diversidad alfa y beta}

La diversidad Alfa de la comunidad de epífitas es baja (Tabla 3). El índice de Shannon arrojó un valor de 0.94, el índice de dominancia de Simpson obtuvo un resultado similar (0.59). En el caso de la diversidad Beta, el coeficiente de Sørensen indicó que la similitud florística entre el transecto y la parcela es baja. Esto podría explicarse por la diferencia de tamaño entre las áreas de muestreo, ya que la del transecto es mucho mayor a la parcela.

\section{Tabla 3. Índices de diversidad Alfa y Beta}

\begin{tabular}{|l|l|}
\hline Índice & Valor arrojado \\
\hline Índice de dominancia de Simpson & 0.59 \\
\hline Índice de Shannon & 0.94 \\
\hline Coeficiente de Similitud de Sorensen & 0.3 \\
\hline
\end{tabular}




\section{Cristopher Jiménez-Orozco, Berleni V. Lebrón-Liriano, Rosy Fernández-Gutiérrez, \\ Rumarda Urbáez y Ángela Guerrero}

\section{Discusión y conclusión}

La flora epífita vascular del Parque Iberoamérica está compuesta por 35 especies. De estas, 26 se encontraron en el transecto, mientras que en la parcela solo se registraron 9. Las Bromeliaceae (Tillandsia recurvata y T. balbisiana) fueron las más abundantes. Estos resultados coinciden con los obtenidos en otros estudios realizados en bosques tropicales (Gentry \& Dodson, 1987; Benzing, 1990), donde se encontró que las bromelias constituyen uno de los grupos de plantas más conspicuos en las comunidades de epífitas del dosel arbóreo. En República Dominicana, Tillandsia es el género de bromelias más diverso y en el que se reporta la mayor cantidad de especies con hábito epífito (Ruiz-Vargas et al., 2015). Un aspecto importante a considerar sobre la biología de estas especies es la forma de reproducción, se ha documentado que algunas epífitas en respuesta a la escasez de nutrientes y humedad han desarrollado mecanismos de reproducción asexual (Benzing, 1987). Durante el trabajo de campo no se encontró evidencia de producción de frutos o flores, lo que sugiere que estas plantas podrían presentar dichas adaptaciones, además cabe resaltar que el área en cuestión está localizada en una zona muy urbanizada dentro de la ciudad.

De acuerdo al inventario de plantas autóctonas y endémicas realizado por Peguero \& García (2015) en la ciudad de Santo Domingo, la única especie epífita presente en el Distrito Nacional es Tillandsia usneoides, la cual ha sido cultivada por su potencial artesanal u ornamental, lo que quizás ha contribuido a su dispersión en sitios antropizados. En el caso de T. recurvata y T. balbisiana se desconoce si son cultivadas, aunque esto podría explicar adecuadamente su presencia en ciertas áreas de la ciudad.

El género Ficus (Moraceae) fue el más diverso de la comunidad de epífitas con cuatro especies (F. citrifolia, F. crassinervia, Ficus crocata y F. microcarpa), siendo $F$. crassinervia y F. microcarpa las de mayor ocurrencia con una abundancia relativa de 58 y 50 individuos, respectivamente. Cabe destacar que en el área de estudio se han identificado nueve especies del género Ficus (Jiménez \& Lebrón-Liriano, 2018).

En otros parques urbanos de la ciudad de Santo Domingo también se han estudiado las especies de Ficus, tal es el caso del Parque Mirador Sur (Agüero, 2014). El referido estudio consistió en una caracterización de las poblaciones de tres especies nativas del género (F. citrifolia, F. crocata y F. crassinervia), donde se las reporta como especies de hábito principalmente rupícola o terrestre. Sin embargo, dichos resultados difieren con los obtenidos en este trabajo, ya que las especies en cuestión presentaron predominantemente hábito epífito. Estos datos tampoco coinciden con la descripción botánica de las especies, ya que las mismas son descritas como rupícolas o terrestres (Piedra, Ramírez, \& Ibarra, 2006; Ibarra, Cornejo, González, Piedra \& Luna, 2012). Esta variación es un indicador de la alta plasticidad morfológica que exhiben las especies del género.

En cuanto a la relación epífita-forófito se encontró poca especificidad por parte de las epífitas. Nephrolepis acutifolia (Nephrolepidaceae) fue la única especie que se observó creciendo sobre Phoenix dactylifera (Arecaceae). Sin embargo, no hay estudios que respalden este tipo de relación. En general, este fenómeno es poco común, especialmente en plantas con tanta plasticidad como las epífitas (Vergara, Pacheco \& Flores, 2010) y es atribuido a las características estructurales o morfológicas de los forófitos (Laube \& Zotz, 2006; Callaway, Reinhart, Moore, Moore, \& Pennings, 2002; Merwin, Rentmeester \& Nadkarni, 2003).

La ausencia de ciertos taxa como las Orchidaceae, que a menudo conforman más del $50 \%$ de la diversidad de especies de epífitas vasculares en los bosques tropicales (Krömer \& Gradstein, 2003), y la baja ocurrencia de grupos como las pteridofitas (helechos), se debe al alto grado de perturbación 
antrópica y la contaminación a los que está expuesto el parque. A esto se suma, el mal manejo por parte de las autoridades en materia ambiental.

\section{Recomendaciones}

Recomendamos que se realicen estudios de esta naturaleza en otros parques urbanos de Santo Domingo. Además de monitoreos a las comunidades estudiadas con la finalidad de registrar cambios en la composición y distribución de las especies. La información levantada en esta investigación servirá de base para la creación de un plan de manejo de la flora en áreas verdes urbanas.

\section{Agradecimientos}

Agradecemos a los miembros de ADEBIO: Adalberto Martínez, Rossé Féliz y Jeremy Then por su apoyo y colaboración, así como también por el préstamo de equipos durante el trabajo de campo para llevar a cabo este proyecto. A Víctor Cabral por la traducción del resumen al inglés y a las maestras Jackeline Salazar y Jeannette Mateo por su asistencia y corrección.

\section{Referencias}

Ackerman, J. D., Montalvo, A. M., \& Vera, A. M. (1989). Epiphyte host specifity of Encyclia krugii, a Puerto Rican endemic orchid. Lindleyana, 4(2): 74-77.

Agüero, M. (2014). Estudio poblacional del género Ficus (L.) en el Parque Mirador del Sur, prov. Santo Domingo, D. N. República Dominicana. (Tesis de licenciatura). Universidad Autónoma de Santo Domingo. Santo Domingo, República Dominicana.

Benzing, D. H. (1987). Vascular epiphytism: Taxonomic participation and adaptive diversity. Annals of the Missouri Botanical Garden, 74(2): 183-204.

Benzing, D. H. (1990). Vascular epiphytes: general biology and related biota. Cambridge, Reino Unido: Cambridge University Press.
Bruijnzeel, L., \& Hamilton, L. S. (2001). Tiempo decisivo para las selvas de neblina. IHP Programa Trópicos Húmedos, (13): 37.

Callaway, R., Reinhart, K., Moore, G., Moore, D. \& Pennings, S. (2002). Epiphyte host preferences and host traits: mechanisms for species-specific interactions. Oecologia, 132(2): 221-230.

Freiberg, M. \& Freiberg, E. (2000). Epiphyte diversity and biomass in the canopy of lowland and montane forests in Ecuador. Journal of Tropical Ecology, 16: 673-688.

García-González, A., Riverón-Giró, F. B., GonzálezRamírez, I. S., Escalona Domenech, R. Y., Hernández Montero, Y. \& Palacio Verdecia, E. (2016). Ecología y estructura poblacional del endemismo cubano Tetramicra malpighiarum (Orchidaceae), en el Parque Nacional Desembarco del Granma, Cuba. Lankesteriana, 16(1): 1-11.

Gentry, A. H., \& Dodson, C. H. (1987). Diversity and biogeography of neotropical vascular epiphytes. Annals of the Missouri Botanical Garden, 74: 205-233.

Gradstein, R., Nadkarni, N., Krömer, T., Holz, I., \& Nöske, N. (2003). A protocol for rapid and representative sampling of vascular and non- vascular epiphytes diversity of Tropical rain forests. Selbyana, 24(1): 105-111.

Granado, L., \& García-Beltrán, J. A. (2016). Requerimientos de microhábitat de las epífitas vasculares en la zona de conservación de la Reserva Florística Manejada Lomas de Galindo, Mayabeque. Revista del Jardin Botánico Nacional, 37: 39-46.

Granados-Sánchez, D., López-Ríos, G. F., HernándezGarcía, M. A., \& Sánchez-González, A. (2003). Ecología de las plantas epífitas. Revista Chapingo Serie Ciencias Forestales y del Ambiente, 9: 101-111.

Greeney, H. F. (2001). The insects of plant-held waters: a review and bibliography. Journal of Tropical Ecology, 17(2): 241-260.

Hager J., \& Zanoni, T. (1993). La Vegetación Natural de la República Dominicana una nueva clasificación. Moscosoa, 7: 39-81. 


\section{Cristopher Jiménez-Orozco, Berleni V. Lebrón-Liriano, Rosy Fernández-Gutiérrez, \\ Rumarda Urbáez y Ángela Guerrero}

Ibarra-Manríquez, G., Cornejo-Tenorio, G., GonzálezCastañeda, N., Piedra-Malagón, E. M., \& Luna, A. (2012). El género Ficus L. (Moraceae) en México. Botanical Sciences, 90(4): 389-452.

Jiménez-Orozco, C., \& Lebrón-Liriano, B. V. (2018). "Estudio poblacional del género Ficus (L.) en el Parque Iberoamérica, Prov. Santo Domingo, D. N. República Dominicana”. En: P. F. GómezRamírez., S. J. Incháustegui., J. F. Guillén., W. Camilo Reynoso., \& A. Núñez-Sellés (Eds.). Libro de Resúmenes del III Congreso Estudiantil de Investigación Cientifica y Tecnológica. (p. 127). Santo Domingo, República Dominicana

Kelly D. L. (1985). Epiphytes and climbers of a Jamaican rain forest: vertical distribution, life forms and life histories. Journal of Biogeography, 12: 223-241.

Krebs, C. J. (1999). Ecological Methodology. 2nd ed. Menlo Park, EE. UU: Benjamin Cummings.

Krömer, T., \& Gradstein, R. (2003). Species richness of vascular epiphytes in two primary forest and fallows in the Bolivian Andes. Selbyana, 24: 190-195.

Laube, S., \& Zotz, G. (2006). Neither hostspecific nor random: vascular epiphytes on three tree species in a Panamanian lowland forest. Annals of Botany, 97(6): 1103-1114.

Llinás, R. A. (2005). Sintesis sobre la situación geológica de la Hispaniola. Santo Domingo, República Dominicana: Ministerios de Minería de las Américas, Ministerio de Estado de Industria y Comercio.

Merwin, M. C., Rentmeester, S. A., \& Nadkarni, N. M. (2003). The influence of host tree species on the distribution of epiphytic Bromeliads in experimental monospecific plantations, La Selva, Costa Rica. Biotropica, 35(1): 37-47.

Migenis L. E., \& Ackerman J. D. (1993). Orchidphorophyte relationship in a forest watershed in Puerto Rico. Journal of Tropical Ecology, 9: 231-240.

Moreno C. (2001). Modelos para medir la Biodiversidad. M\&T, Manuales y Tesis SEA, 1: 84.
Nieder, J. Ibisch, P. L., \& Barthlot, W. (1997). Biodiversidad de Epífitas: una cuestión de escala. La Habana, Cuba. Revista del Jardin Botánico Nacional, 18: 9-62.

Nieder, J., Engwald, S., \& Barthlott, W. (1999). Patterns of neotropical epiphyte diversity. Selbyana, 20: 66-75.

Oficina Nacional de Meteorología (ONAMET). (2019). www.onamet.gob.do. Consultado el 24 de febrero del 2019.

Peguero, B. \& García, R. (2015). Plantas endémicas y nativas cultivadas en la ciudad de Santo Domingo. Moscosoa 19: 96-128.

Piedra-Malagón, E. M., Ramírez, R., \& IbarraManríquez, G. (2006). El género Ficus (Moraceae) en el Estado de Morelos, México. Acta Botánica Mexicana, 75: 45-75.

Ruiz-Vargas, N., Ortiz, C., \& Guerrero, A. (2015). Distribución de la familia Bromeliaceae Juss. en La Española en base a colecciones de herbario. Moscosoa, 19: 22-36.

Smith, R. L., \& Smith, T. M. (2001). Ecología. 4ta ed. Madrid, España: Pearson Education S.A.

The Plant List. (2013). Version 1.1. http://www. theplantlist.org/. Recuperado de: http://www. theplantlist.org/ (consultado febrero 2019).

Tropicos.org. Missouri Botanical Garden. (2010). http://www.tropicos.org. Recuperado de https:// www.tropicos.org/home.aspx?langid=66 (consultado febrero 2019).

Vergara-Torres, C. A., Pacheco-Álvarez, M. C., \& Flores-Palacios, A. (2010). Host preference and host limitation of vascular epiphytes in a tropical dry forest of central Mexico. Journal of Tropical Ecology, 26(6): 563-570.

Wester, S., Mendieta-Leiva, G., Nauheimer, L., Wanek, W., Kreft, H., \& Zotz, G. (2011). Physiological diversity and biogeography of vascular epiphytes at Río Changuinola, Panama. Flora-Morphology, Distribution, Functional Ecology of Plants, 206(1): 66-79.

Wolf, J. H. D. \& Flamenco, A. (2003). Patterns in species richness and distribution of vascular epiphytes in Chiapas, Mexico. Journal of Biogeography, 30: 1689-1707. 
Zotz, G. \& Schultz, S. (2008). The vascular epiphytes of a lowland forest in Panama-species composition and spatial structure. Plant Ecology 194(1): 131-141.
Zotz, G., \& Hietz, P. (2001). The physiological ecology of vascular epiphytes: current knowledge, open questions. Journal of Experimental Botany 52: 2067-2078. 